\title{
Is irradiation significantly associated with a higher risk for CVD?
}

\author{
Mohammad Hossein Panahi ${ }^{1} \cdot$ Razieh Bidhendi Yarandi $^{1,2}$ (D)
}

Received: 1 April 2019 / Accepted: 16 April 2019 / Published online: 25 April 2019

(c) Springer-Verlag GmbH Germany, part of Springer Nature 2019

Keywords Statistical issues $\cdot$ Testimation bias $\cdot$ Sparse data bias

\section{Dear Editor,}

We read with great interest the recent paper by Yu-Chun Yeh et.al published in the European Archives of OtoRhino-Laryngology Journal entitled "The effectiveness of high-resolution ultrasound in the assessment of the carotid intima-media thickness for post irradiated neck" [1].

The aim of the paper was to investigate the risk factors for an increased mean carotid intimal-medial thickness CIMT which is a strong predictor of future cardiovascular events. We think some methodological issues should be considered to avoid misleading interpretation of the results. First, they declared that all statistically significant variables in the univariate analysis were selected for inclusion in the multivariate linear regression. It was proved that the estimated coefficient through this approach would be subjected to testimation bias. To avoid this issue, selection of variables with more liberal levels, such as $p$ value $<0.20$ or $p$ value $<0.50$ and other methods such as directed acyclic graph (DAG) and new approaches such as bootstrap and Bayesian model averaging could be helpful [2-4]. Second, univariate and multivariate logistic regression models were performed to investigate the risk for an abnormal mCIMT result. The authors declared they find the previous irradiation as a factor that is strongly associated with an increased CIMT after controlling for multiple associated factors, such as DM, hypertension, hyperlipidemia, and

This reply refers to the comment available at https://doi. org/10.1007/s00405-019-05302-1.

Razieh Bidhendi Yarandi razi_bidhendi@yahoo.com

1 Department of Epidemiology and Biostatistics, School of Public Health, Tehran University of Medical Sciences, Tehran, Iran

2 Reproductive Endocrinology Research Center, Research Institute for Endocrine Sciences, Shahid Beheshti University of Medical Sciences, Tehran, Iran smoking (OR 13.5, 95\% CI 1.48-122.8). Actually, a big odds ratio does not always mean a strong predictor. A big odds ratio with a wide confidence interval could be due to the sparse data problem leading to biased estimation of parameters. This bias occurs when observations in the combination of exposure and outcome strata are rare. The authors could use appropriate statistical methods such as Firth penalization and data augmentation to reduce sparse data bias $[5,6]$. As a conclusion, we think that due to the bias estimation of parameters, the authors' claims regarding evidence that irradiation was significantly associated with a higher risk for CVD would be unreliable.

Acknowledgements This research received no specific grant from any funding agency in the public, commercial, or not-for-profit sectors.

\section{Compliance with ethical standards}

Conflict of interest The authors declare that they have no conflict of interest.

\section{References}

1. Yeh YC et al (2019) The effectiveness of high-resolution ultrasound in the assessment of the carotid intima-media thickness for postirradiated neck. Eur Arch Oto-Rhino-Laryngol 276(4):1167-1173

2. Jewell NP (2003) Statistics for epidemiology. Chapman and Hall, London

3. Steyerberg EW (2008) Clinical prediction models: a practical approach to development, validation, and updating. Springer Science \& Business Media, New York

4. Rothman KJ, Greenland S, Lash TL (2008) Modern epidemiology, vol 3. Wolters Kluwer Health, Philadelphia

5. Heinze G, Schemper M (2002) A solution to the problem of separation in logistic regression. Stat Med 21(16):2409-2419

6. Firth D (1993) Bias reduction of maximum likelihood estimates. Biometrika 80(1):27-38

Publisher's Note Springer Nature remains neutral with regard to jurisdictional claims in published maps and institutional affiliations. 